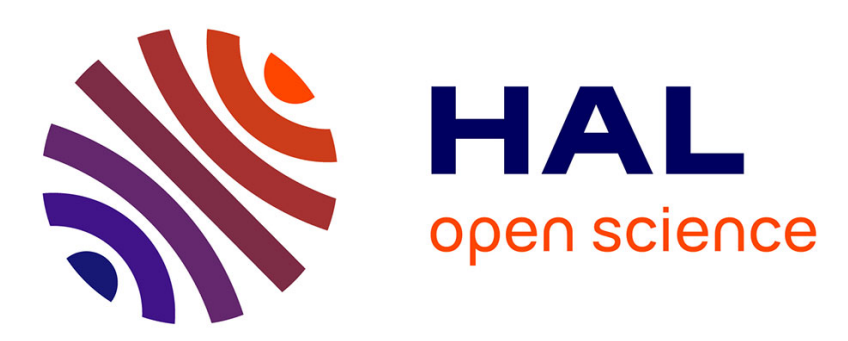

\title{
Comparaison de deux bobinages pour la propulsion d'un véhicule hybride urbain
}

\author{
Hoang Cong Minh Mai, Fouad Charih, Frédéric Dubas, Didier Chamagne, \\ Christophe Espanet
}

\section{- To cite this version:}

Hoang Cong Minh Mai, Fouad Charih, Frédéric Dubas, Didier Chamagne, Christophe Espanet. Comparaison de deux bobinages pour la propulsion d'un véhicule hybride urbain. European Journal of Electrical Engineering (EJEE), 2011, 14 (2-3), pp.255 - 285. 10.3166/Geo.19.11-38 . hal-02300080

\section{HAL Id: hal-02300080 \\ https://hal.science/hal-02300080}

Submitted on 29 Sep 2019

HAL is a multi-disciplinary open access archive for the deposit and dissemination of scientific research documents, whether they are published or not. The documents may come from teaching and research institutions in France or abroad, or from public or private research centers.
L'archive ouverte pluridisciplinaire HAL, est destinée au dépôt et à la diffusion de documents scientifiques de niveau recherche, publiés ou non, émanant des établissements d'enseignement et de recherche français ou étrangers, des laboratoires publics ou privés. 
archives-ouvertes

\title{
Comparaison de deux bobinages pour la propulsion d'un véhicule hybride urbain
}

\author{
Hoang Cong Minh Mai, Fouad Charih, Frédéric Dubas, Didier Chamagne, \\ Christophe Espanet
}

\section{To cite this version:}

Hoang Cong Minh Mai, Fouad Charih, Frédéric Dubas, Didier Chamagne, Christophe Espanet. Comparaison de deux bobinages pour la propulsion d'un véhicule hybride urbain. European Journal of Electrical Engineering (EJEE), 2011, 14 (2-3), pp.255 - 285. hal-02300080

\section{HAL Id: hal-02300080 \\ https://hal.archives-ouvertes.fr/hal-02300080}

Submitted on 29 Sep 2019

HAL is a multi-disciplinary open access archive for the deposit and dissemination of scientific research documents, whether they are published or not. The documents may come from teaching and research institutions in France or abroad, or from public or private research centers.
L'archive ouverte pluridisciplinaire HAL, est destinée au dépôt et à la diffusion de documents scientifiques de niveau recherche, publiés ou non, émanant des établissements d'enseignement et de recherche français ou étrangers, des laboratoires publics ou privés. 


\title{
Comparaison de deux bobinages pour la propulsion d'un véhicule hybride urbain
}

\author{
Hoang Cong Minh Mai — Fouad Charih — Frédéric Dubas - \\ Didier Chamagne - Christophe Espanet \\ Laboratoire FEMTO-ST, Département ENISYS, Université de Franche-Comté \\ Parc Technologique, 2 avenue Jean Moulin, F-90010 Belfort, France \\ hcmmai@univ-fcomte.fr, fcharih@edu.univ-fcomte.fr, FDubas@gmail.com, \\ didier.chamagne@univ-fcomte.fr, christophe.espanet@univ-fcomte.fr
}

RÉSUMÉ. L'utilisation de machines synchrones à aimants permanents (MSAP) pour la réalisation de moteurs intégrés dans les roues d'un véhicule hybride urbain est étudiée dans cet article en considérant deux types de bobinage (i.e., à pas diamétral et à pas dentaire, bobiné autour d'une dent). Différentes grandeurs électromagnétiques telles que la résistance, les inductances, les pertes fer, les pertes dans les aimants, les pertes Joule et les forces magnétiques sur les dents sont calculées afin de comparer les performances des deux machines. L'étude montre l'intérêt d'utiliser un bobinage à pas dentaire par rapport à un bobinage à pas diamétral : le couple de détente est réduit, le taux de distorsion harmonique de la f.é.m. à vide est plus faible, les pertes Joule sont plus faibles et le défluxage de la machine est facilité par une inductance de phase plus élevée. Il convient toutefois de noter les inconvénients du bobinage concentré tels que les pertes dans les aimants et la variation des forces magnétiques auxquelles sont soumises les dents du stator. Ces effets non désirés sont dus aux harmoniques du champ magnétique engendrés par la distribution spatiale du bobinage dentaire.

ABSTRACT. The use of permanent magnet synchronous machines (PMSM) for in-wheel electrical motorization of an urban hybrid vehicle has been studied in this article. Two types of winding, distributed and concentrated winding around a tooth, have been considered for the winding of the PMSM. Different electromagnetic sizes such as the resistance, the inductances, the iron losses, the PM eddy-current losses and teeth magnetic forces have been considered for the comparison of the two machines performances. The study shows the advantages of concentrated winding in comparison with the distributed winding, such as the diminution of the cogging torque, the reduction of no-load emf harmonics and the increase of the flux weakening range due to the increase of the inductance. However, it is important to notice the drawbacks of concentrated winding: the high level of PM eddy-current losses and large variation of stator teeth magnetic forces. These undesired effects are due to the harmonics of magnetic field produced by the concentrated windings.

MOTS-CLÉS : Machine synchrone à aimants permanents (MSAP), bobinages distribués, bobinages concentrés, pertes Joule, pertes dans les aimants, rendement du moteur, forces magnétiques.

KEYWORDS: Permanent magnet synchronous machine (PMSM), distributed windings, fractional slot, concentrated windings, copper losses, PM eddy-current losses, motor efficiency, magnetic forces.

DOI:10.3166/Geo.19.11-38

Revue. Volume $\mathrm{X}-\mathrm{n}^{\circ} \mathrm{x} /$ année, pages 1 à $\mathrm{X}$ 


\section{Introduction et contexte de l'étude}

Dans le cadre du développement d'un véhicule hybride de type urbain, les auteurs ont étudié la conception de moteur-roues électriques de type synchrone à aimants permanents pour la motorisation électrique du véhicule. Le véhicule hybride est de type quadricycle avec une masse totale en charge de moins d'une tonne.

La structure du véhicule hybride est simple. Il est constitué d'un moteur thermique classique qui permet la traction du véhicule par le train avant. Deux moteurs électriques sont également intégrés dans les deux roues du train arrière permettant la propulsion électrique du véhicule. Le couplage des puissances électriques et thermiques est donc effectué par le sol. L'alimentation électrique est assurée par un pack énergie/puissance constitué d'une batterie et de supercondensateurs, le tout étant intégré dans le plancher du véhicule. Le véhicule possède également un chargeur pour recharger les batteries depuis un réseau d'énergie électrique dans une station ou à domicile. Les deux moteurs électriques sont alimentés par le pack énergie/puissance via deux onduleurs et tout le système est contrôlé par une électronique de gestion d'énergie, le « cœur ». Cette structure du véhicule est compacte et permet à la fois de fonctionner comme une voiture thermique classique, et donc d'en posséder les avantages en terme d'autonomie, mais aussi de fonctionner en mode électrique afin d'économiser du carburant et de limiter les émissions de polluants.

Concernant les moteur-roues électriques du véhicule, ils sont de type synchrone à aimants permanents avec des aimants montés en surface. Pour faciliter l'intégration du moteur dans la roue, ce dernier est à structure inversée (i.e., à rotor extérieur). Dans ce contexte, nous nous proposons d'étudier et de comparer deux machines : une première est une machine à nombre d'encoche par pôle et par phase nonfractionnaire (ou machine non-fractionnaire) utilisant un bobinage distribué de type diamétral et une seconde est une machine à nombre d'encoche par pôle et par phase fractionnaire (ou machine fractionnaire) avec un bobinage concentré autour des dents (de type dentaire ou encore à pas dentaire).

Concernant la première machine, son existence est connue depuis longtemps dans l'industrie et son utilisation est classique. Ce type de bobinage permet d'enlacer un maximum de flux magnétique dans chaque phase. En revanche, un défaut important du type de bobinage employé est lié à l'encombrement axial des têtes des bobines et au fait que les bobines sont entrelacées. Ces défauts conduisent d'une part à des difficultés de réalisation du bobinage (l'automatisation est impossible à réaliser avec un taux de remplissage acceptable) mais également d'autre part à l'augmentation des pertes Joule. Dans le contexte de l'intégration du moteur dans une roue, l'encombrement axial est clairement une contrainte limitante, de même que les échauffements (en particulier du fait de la proximité du système de freinage mécanique). 
Comparaison de deux types de bobinage pour la réalisation de moteur-roues à aimants permanents destinés à la propulsion d'un véhicule hybride urbain

La deuxième machine (i.e., fractionnaire à bobinage dentaire) a été très largement étudiée car les têtes de bobines sont fortement réduites, ce qui permet de réduire les pertes Joule, et la réalisation peut être relativement facilement automatisée (Bianchi et al., 2006, Chan et al., 1994, Cros et al., 2002). Une machine fractionnaire à bobinage concentré est ainsi potentiellement plus compacte dans la direction axiale qu'une machine classique à bobinage distribué. Pour la conception de la machine, plusieurs combinaisons de nombre de pôles par rapport au nombre d'encoches sont possibles (Libert, 2004). Pour certaines configurations, la machine peut avoir un très faible couple de détente et, suivant le type de bobinage, un contenu harmonique de la f.é.m plus favorable.

Malgré ces avantages, le champ magnétique d'entrefer créé par ce type de bobinage présente plus d'harmoniques spatiaux, et cela peut entraîner des effets nondésirés, comme par exemple la saturation locale du circuit magnétique, d'importantes variations des forces magnétiques auxquelles sont soumises les dents ou bien encore d'intenses pertes par courants Foucault dans les aimants.

Dans ce contexte, les auteurs présentent tout d'abord la structure du véhicule hybride considéré dans cette étude et le cahier des charges pour les moteurs électriques intégrés dans les roues arrière. Ils définissent ensuite les conditions de comparaison de deux machines, l'une utilisant un bobinage à pas diamétral et l'autre un bobinage à pas dentaire. S'en suit une comparaison de plusieurs grandeurs électromagnétiques clés : les forces électromotrices à vide, le couple de détente, les pertes Joule, les pertes magnétiques dans le stator et les pertes par courant de Foucault dans les aimants permanents. Un paragraphe traite ensuite plus particulièrement des comparaisons des inductances et de l'influence sur la capacité de défluxage des deux machines. Enfin, les auteurs s'intéressent à la comparaison des forces magnétiques auxquelles sont soumises les dents.

\section{Véhicule hybride et cahier des charges du moteur roue électrique}

\subsection{Synoptique général du véhicule}

La Figure 1 représente la structure générale du véhicule avec les différents organes des motorisations thermique et électrique : le moteur thermique, les moteurs électriques, les convertisseurs et le pack batteries/super-condensateurs. Le véhicule peut fonctionner en mode tout thermique en zone extra-urbaine ou en mode tout électrique (fonctionnement du véhicule à zéro émission (ZEV)) en ville. La présence de la motorisation électrique permet de réduire le dimensionnement du moteur thermique, la puissance maximale étant obtenue par un fonctionnement simultané du moteur thermique et des deux moteurs électriques. Dans l'application considérée, le véhicule doit pouvoir fonctionner en mode tout électrique en pleine charge pendant $70-80 \mathrm{~km}$, avec une vitesse maximale de $50 \mathrm{~km} / \mathrm{h}$. La récupération d'énergie doit également être possible au freinage : pendant cette phase, les machines électriques 
fonctionnent en mode génératrice pour recharger le pack énergétique constitué des batteries et des supercondensateurs.

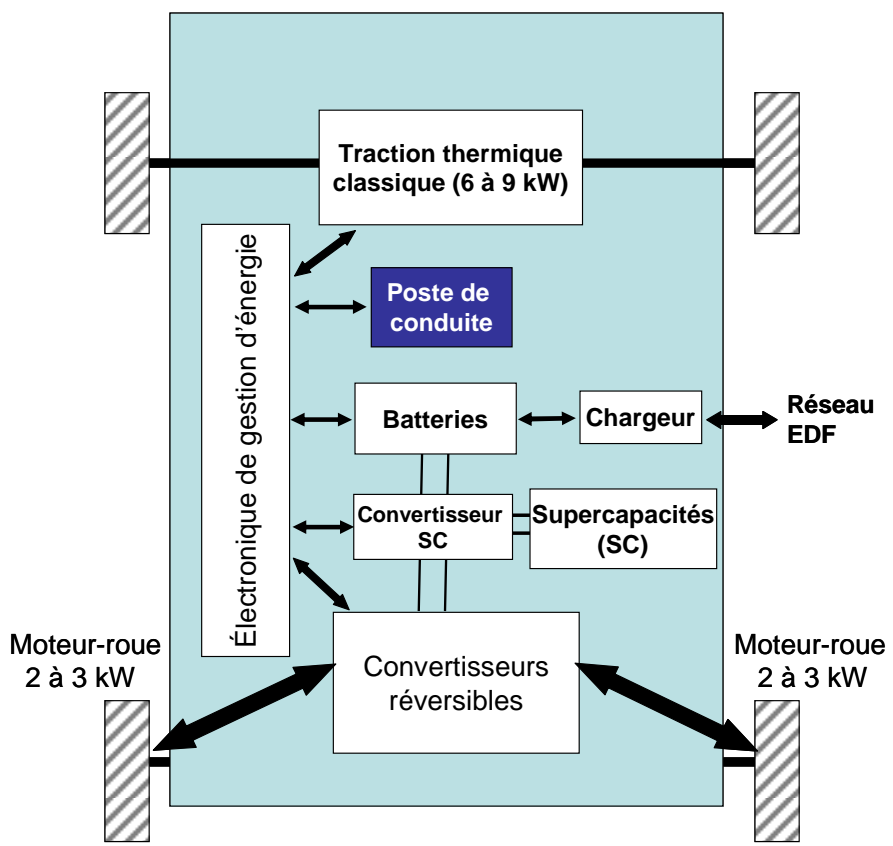

Figure 1. Synoptique de la structure générale du véhicule.

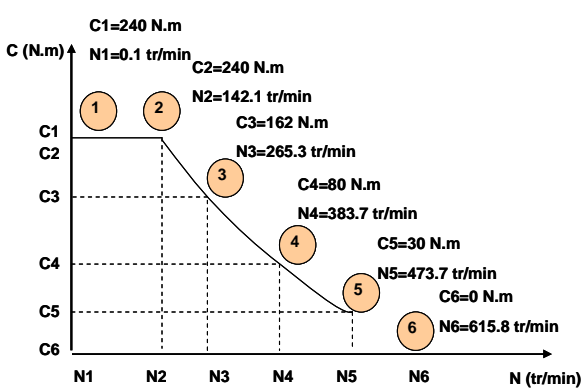

a) Couple-Vitesse

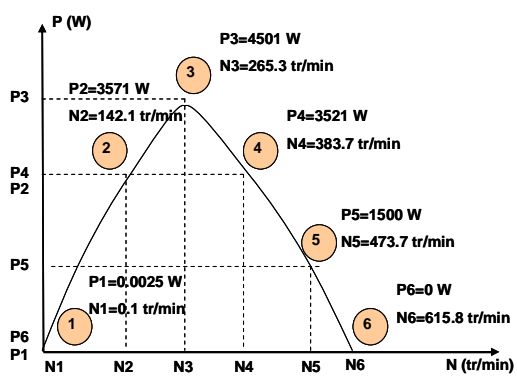

b) Puissance-Vitesse

Figure 2. Caractéristiques du moteur-roue (Cahier des charges).

\subsection{Cahier des charges du moteur-roue électrique}

La Figure 2 présente les caractéristiques couple-vitesse et puissance-vitesse de chaque moteur-roue électrique, ces caractéristiques étant obtenues à partir du cahier 
Comparaison de deux types de bobinage pour la réalisation de moteur-roues à aimants permanents destinés à la propulsion d'un véhicule hybride urbain

des charges fonctionnel du véhicule. La courbe comporte 6 points de fonctionnement que le moteur doit impérativement réaliser. À chaque point de fonctionnement est associé un couple que le moteur doit produire et une vitesse de rotation imposée par la vitesse du véhicule et le périmètre de la roue. Dans l'application étudiée, le moteur est en attaque directe, c'est-à-dire qu'il n'y a pas de réducteur entre le moteur et la roue. L'adjonction d'un réducteur permettrait de réduire le volume et la masse du moteur (grâce à la réduction du couple à fournir), mais l'intégration du réducteur poserait des difficultés mécaniques et atténuerait le gain en volume obtenu par la réduction du couple à fournir.

La courbe caractéristique de la Figure 2 est déduite à partir d'une analyse complète du comportement routier du véhicule. Les points 1 et 2 correspondent à un fonctionnement à couple maximal (démarrage en côte et accélération maximale). Le point 3 correspond au point à puissance maximale. Le point 4 correspond au couple thermique (c'est-à-dire au régime thermique moyen couramment appelé service $\mathrm{S} 1$ ). Le point 5 correspond au fonctionnement en mode tout électrique à vitesse maximale $(50 \mathrm{~km} / \mathrm{h}$ au niveau du véhicule). Et enfin le point 6 correspond à un fonctionnement en mode thermique à la vitesse maximale de $65 \mathrm{~km} / \mathrm{h}$; dans ces conditions, la machine électrique est entraînée et il ne faut pas qu'elle débite dans la batterie via les diodes de roue libre de l'onduleur de tension. Ce point 6 est également un point de référence pour étudier les pertes fer et les pertes dans les aimants permanents lorsque la machine est entrainée en génératrice : ces pertes ne doivent pas être trop élevées pour limiter le freinage des moteur-roues en mode thermique.

\section{Conditions de comparaison}

Deux machines avec deux types de bobinage ont été dimensionnées pour la motorisation électrique du véhicule. Les deux machines ont été conçues avec un outil d'optimisation (le logiciel Pro@design de la société 'Design Processing Technologies') afin d'obtenir une masse minimale tout en vérifiant des contraintes sur l'échauffement adiabatique à couple maximal et sur les pertes magnétiques à vitesse maximale. Les deux machines ont le même nombre de pôles afin de se placer dans des conditions de comparaison les plus cohérentes possibles.

Pour la machine ayant un bobinage concentré, nous choisissons une configuration avec une combinaison de nombre de dents $N d$ et de nombre de pôles $2 p$ telle que : $N d=2 p \pm 2$. Ce choix permet d'obtenir un facteur de bobinage élevé (Ishak, 2004, Libert, 2004). On précise également que toutes les dents de la machine sont bobinées (i.e., bobinage à double couche).

La machine non-fractionnaire avec un bobinage à pas diamétral, appelée machine M1, est alimentée avec des courants en créneaux dans la mesure où les forces électromotrices sont approximativement trapézoïdales. Ce type d'alimentation ne permet pas d'augmenter la vitesse par déphasage du courant par rapport aux forces électromotrices (défluxage) car l'application de courants en créneaux fortement 
déphasés par rapport aux forces électromotrices provoque de fortes ondulations de couple. La machine fractionnaire avec un bobinage à pas dentaire, appelée machine M2, est alimentée quant à elle par des courants quasi- sinusoïdaux dans la mesure où les forces électromotrices sont sinusoïdales. Afin de comparer les performances des machines M1 et M2, nous calculons différentes grandeurs électromagnétiques en utilisant des modèles analytiques et des modèles numériques reposant sur la méthode des éléments finis bidimensionnels (2D).

La Figure 3 donne les coupes transversales des deux machines M1 et M2. Les Tableaux 1 et 2 donnent respectivement les principaux paramètres et les différentes masses des parties actives des deux machines M1 et M2.

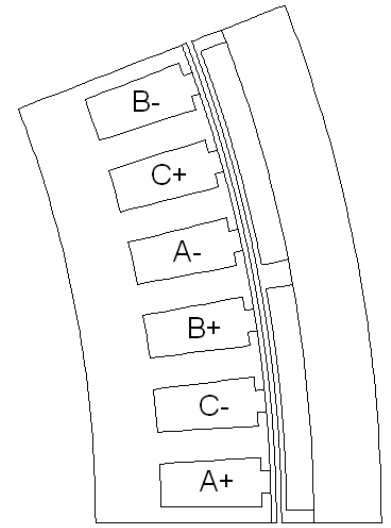

a) $M 1$ (2 pôles)

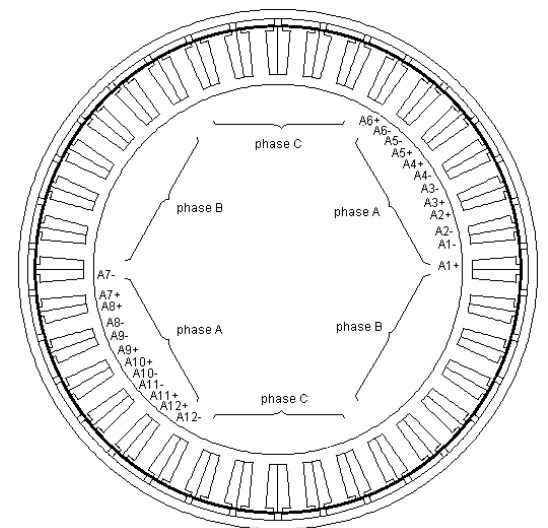

b) M2 (complète)

Figure 3. Coupe transversale et bobinage des deux machines.

Tableau 1. Paramètres géométriques et magnétiques des deux machines.

\begin{tabular}{lcc} 
Paramètres & M1 & M2 \\
\hline Rayon extérieur du rotor, $R_{\text {ext }}[\mathrm{mm}]$ & 155 & 155 \\
Angle d'ouverture des aimants, $\alpha_{a}[\mathrm{rad}]$ & 0,9 & 0,86 \\
Induction rémanente des aimants Nd-Fe-B, $B r_{a}[\mathrm{~T}]$ & 1,3 & 1,3 \\
Rayon intérieur du stator, $\mathrm{R}_{\text {int }}[\mathrm{mm}]$ & 126,8 & 111,4 \\
Épaisseur des aimants, $h a[\mathrm{~mm}]$ & 3 & 3,8 \\
Ouverture d'encoche, $\theta_{e}[\mathrm{rad}]$ & 0,017 & 0,105 \\
Entrefer, ent $[\mathrm{mm}]$ & 1,72 & 1,2 \\
Longueurs du fer active et des aimants, $L_{f e r}=L_{\text {aim }}[\mathrm{mm}]$ & 52,4 & 30,7 \\
Nombre de paires de pôles, $p[-]$ & 17 & 17 \\
Nombre des dents, $N d[-]$ & 102 & 36
\end{tabular}


Comparaison de deux types de bobinage pour la réalisation de moteur-roues à aimants permanents destinés à la propulsion d'un véhicule hybride urbain

Tableau 2. Masses des différentes parties actives des deux machines.

\begin{tabular}{lcc} 
Masses & M1 & M2 \\
\hline Masse des aimants, $m_{\text {aim }}[\mathrm{kg}]$ & 1,0 & 0,7 \\
Masse du rotor, $m_{\text {rot }}[\mathrm{kg}]$ & 2,9 & 1,3 \\
Masse du stator, $m_{\text {stat }}[\mathrm{kg}]$ & 2,3 & 0,9 \\
Masse des dents, $m_{d}[\mathrm{~kg}]$ & 1,8 & 2.5 \\
Masse du cuivre, $m_{\text {cu }}[\mathrm{kg}]$ & 1,8 & 1,9 \\
Masse totale du moteur, $m_{\text {tot }}[\mathrm{kg}]$ & 9,8 & 7,4
\end{tabular}

\section{Comparaison des grandeurs électromagnétiques des deux machines étudiées}

\subsection{La f.é.m à vide et le couple de détente}

Les comparaisons de la f.é.m. à vide et du couple de détente sont effectuées à l'aide de simulations par éléments finis en 2D. Les Figures 4 et 5 montrent les chronogrammes des f.é.m ainsi que leur spectre pour une simulation à vide au point 6 (vitesse maximale). La Figure 6 présente l'évolution des couples de détente. Ces figures montrent clairement que la machine M2 (i.e., fractionnaire avec un bobinage dentaire) possède une f.é.m. à vide avec un faible contenu spectral et un couple de détente négligeable devant celui de la machine M1 (i.e., non-fractionnaire avec un bobinage à pas diamétral).

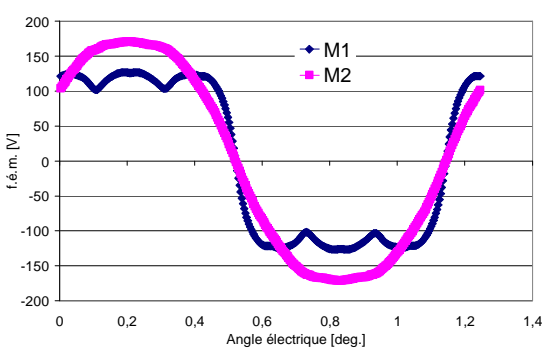

Figure 4. Évolution de la f.é.m. en fonction de la position angulaire $d u$ rotor par rapport au stator à 615,8 $\mathrm{tr} / \mathrm{min}$.

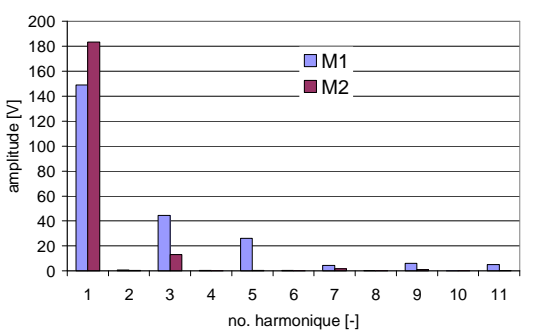

Figure 5. Analyse harmonique de la f.é.m. à vide à $615,8 \mathrm{tr} / \mathrm{min}$. 


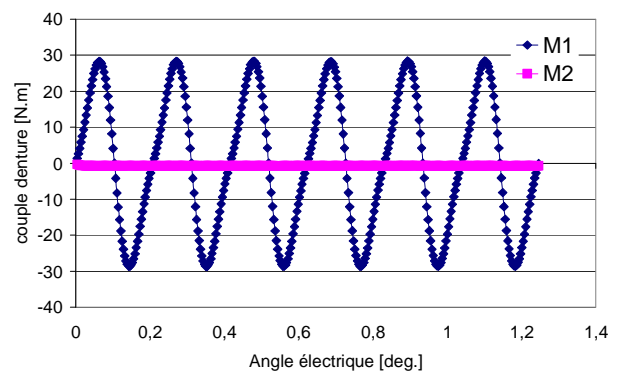

Figure 6. Évolution du couple de détente en fonction de la position angulaire du rotor par rapport au stator à $615,8 \mathrm{tr} / \mathrm{min}$.

\subsection{Résistances statoriques}

Nous nous proposons de calculer dans ce paragraphe les résistances de phase des deux machines étudiées (i.e., M1 et M2). Il s'agit essentiellement d'un problème de calcul géométrique des longueurs de spire.

Dans une bobine, chaque spire de conducteur est décomposée en deux parties : la partie des conducteurs actifs dans l'encoche et la partie de raccordement qui forme les têtes des bobines. De plus, les têtes de bobines se décomposent en deux parties : la partie de fil qui relie deux encoches et la partie tournante du fil qui relie la partie précédente. Pour la machine M1 (i.e., non-fractionnaire avec un bobinage à pas diamétral), en considérant un bobinage en demi-couche, la longueur d'une tête de bobine peut se calculer selon (Espanet, 1999) :

$l_{\text {tbobl }}=l_{\text {sptb1 }}+l_{\text {sptb2 }}$

dans laquelle la longueur de la première partie entre le centre de la partie basse de l'encoche et le centre de la partie haute de l'encoche s'exprime par (si la polarité $p$ est suffisamment élevée) :

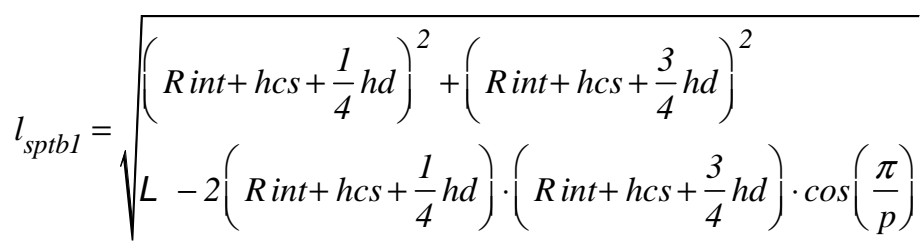

où Rint, hcs, $h d, p$ sont les paramètres géométriques définis dans le Tableau 1.

et la longueur de la partie tournante de tête de bobine s'exprime par: 
Comparaison de deux types de bobinage pour la réalisation de moteur-roues à aimants permanents destinés à la propulsion d'un véhicule hybride urbain

$l_{s p t b 2}=2 \cdot\left[\frac{\pi}{3 \cdot p \cdot q} \cdot\left(\right.\right.$ Rint $\left.\left.+h c s+\frac{1}{2} h d\right)-l d\right]$

où $q$ est le nombre d'encoches par pôle par phase et $l d$ est largeur d'une dent statorique.

Enfin la longueur d'une spire s'exprime par :

$l_{\text {spirel }}=2 \cdot\left(l_{f e r}+l_{t b o b 1}\right)$

Ainsi la résistance d'une phase de la machine M1 (i.e., non-fractionnaire avec un bobinage à pas diamétral) peut se calculer par :

$R_{l}(T c u)=\rho_{C u}\left(T_{C u}\right) \cdot \frac{l_{\text {spirel }}}{S_{\text {cond } 1}} \cdot N_{s p} \cdot p \cdot q$

avec $\rho_{C u}\left(T_{C u}\right)$ la résistivité électrique du cuivre dépendant de la température de fonctionnement du cuivre $T_{C u}, S_{\text {cond } 1}$ la section d'un conducteur pour un bobinage à pas diamétral, $N_{s p}$ le nombre de spires en série dans une encoche.

Pour la machine M2 (i.e., fractionnaire avec un bobinage dentaire), nous proposons un calcul simplifié des têtes des bobines en supposant que la tête de bobine pour chaque spire forme un demi-cercle comme représenté sur la Figure 7. Pour un bobinage en double couche (toutes les dents bobinées), la longueur d'une tête de bobine est donc :

$l_{t b o b 2}=\pi \cdot\left(l d+\frac{b_{o e}}{2}\right)$

où $b_{o e}$ représente l'ouverture d'une encoche.

La longueur d'une spire est alors calculée par :

$l_{\text {spire } 2}=2 \cdot\left(L f e r+l_{\text {tbob } 2}\right)$

et la résistance d'une phase de la machine M2 (i.e., fractionnaire avec un bobinage dentaire) peut se calculer par : 


$$
R_{2}\left(T_{C u}\right)=\rho_{C u}\left(T_{C u}\right) \cdot \frac{l_{\text {spire } 2}}{S_{\text {cond } 2}} \cdot N_{s p} \cdot N_{b m}
$$

avec $S_{\text {cond } 2}$ la section d'un conducteur pour un bobinage à pas dentaire et $N_{b m}$ le nombre de bobines par phase.

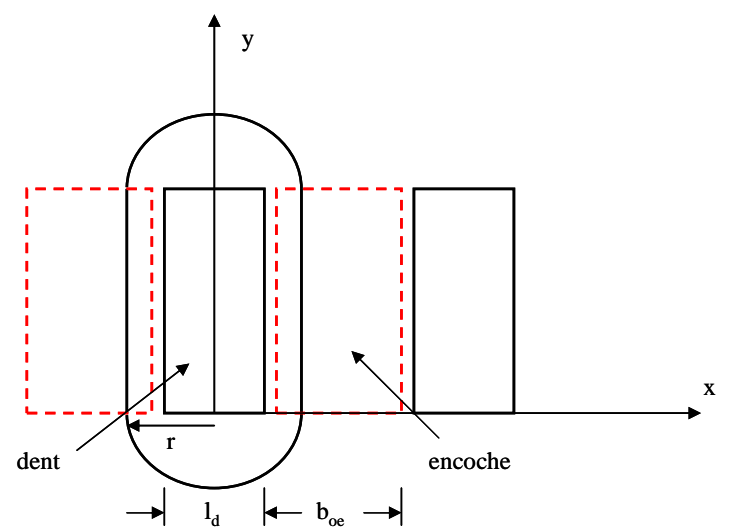

Figure 7. Schéma d'une tête de bobine d'un bobinage concentré.

\subsection{Pertes Joule}

La machine M1 (i.e., non-fractionnaire avec un bobinage à pas diamétral) est alimentée avec des courants en créneaux de largeur $120^{\circ}$ électriques. Les pertes Joule totales s'expriment par :

$P_{J I}\left(T_{C u}\right)=3 \cdot R_{l}\left(T_{C u}\right) \cdot\left(\sqrt{\frac{2}{3}} \cdot I_{l}\right)^{2}=2 \cdot R_{l}\left(T_{C u}\right) \cdot I_{I}^{2}$

avec $I_{1}$ la valeur maximale du courant rectangulaire d'alimentation de la machine M1.

La machine M2 (i.e., fractionnaire avec un bobinage à pas dentaire) est alimentée avec des courants sinusoïdaux. Les pertes Joule totales s'expriment alors par :

$P_{J 2}\left(T_{C u}\right)=3 / 2 R_{2}\left(T_{C u}\right) \cdot I_{2}^{2}$ 
Comparaison de deux types de bobinage pour la réalisation de moteur-roues à aimants permanents destinés à la propulsion d'un véhicule hybride urbain

avec $I_{2}$ la valeur maximale du courant sinusoïdal d'alimentation de la machine M2.

La Figure 8 compare les pertes Joule des deux machines pour les différents points de fonctionnement définis dans le $\$ 2$. On constate que, pour des couples identiques à fournir, les pertes dans le cuivre sont environ $20 \%$ plus importantes avec la machine utilisant un bobinage à pas diamétral. Cette variation est difficile à interpréter car de nombreux paramètres évoluent d'une machine à l'autre. En premier lieu, l'optimisation de la machine à conduit à un entrefer plus faible dans le cas de la machine utilisant un bobinage dentaire $(1,2 \mathrm{~mm}$ au lieu de $1,7 \mathrm{~mm})$ et à une épaisseur d'aimant plus élevée $(3,8 \mathrm{~mm}$ au lieu de $3 \mathrm{~mm})$, ce qui permet d'obtenir une induction dans l'entrefer plus élevée pour la machine à bobinage dentaire $(1,02$ $\mathrm{T}$ au lieu de 0,90 T). Cela permet de réduire les ampères-tours nécessaires pour produire un certain couple dans le cas de la machine à bobinage dentaire. En second lieu, l'optimisation a conduit à une section d'encoche plus importante dans le cas de la machine à bobinage dentaire, mais les volumes de cuivre sont comparables car la longueur de fer est plus faible. Par conséquent la densité efficace de courant est plus faible pour la machine utilisant le bobinage à pas dentaire, ce qui, à volume de cuivre approximativement constant, conduit à des pertes Joules plus faibles. La comparaison est toutefois difficilement généralisable dans la mesure où l'optimisation géométrique globale de la machine a conduit à des entrefers, des longueurs de fer et des sections de cuivre différents.

\subsection{Pertes magnétiques dans les tôles statoriques}

Pour le calcul des pertes magnétiques dans les tôles statoriques, on adopte une approche analytique classique reposant sur la somme des pertes par courant de Foucault et des pertes par hystérésis dans les dents et dans la culasse statorique.

L'expression générale des pertes magnétiques dans les tôles statoriques est :

$$
P_{\text {Fer }}=P_{\text {Hyst }}+P_{\text {Fouc }}=k_{\text {Hyst }} \cdot f \cdot B_{m}^{2}+k_{\text {Fouc }} \cdot f^{2} \cdot B_{m}^{2}
$$

où $B_{m}$ est l'induction magnétique maximale dans la zone considérée du circuit magnétique, $f$ la fréquence électrique de l'induction magnétique, $k_{H y s t}$ et $k_{F o u c}$ sont respectivement les coefficients de calcul des pertes par hystérésis et par courant de Foucault. Ces coefficients sont identifiés pour chaque type de tôle considéré. La somme des pertes fer dans les dents et dans la culasse statorique permet le calcul des pertes fer au stator.

La Figure 9.a donne l'évolution des pertes fer au stator à vide en fonction de la fréquence électrique. On constate que les pertes de la machine M2 (i.e., fractionnaire avec un bobinage dentaire) sont plus faibles. Ceci étant, la Figure 9.b compare les pertes volumiques des deux machines étudiées (environ $10 \%$ plus faibles que la machine M2). Cela s'explique par le fait que les niveaux d'induction sont 
comparables pour les deux machines étudiées (un peu plus faible pour la machine M2) et que le volume de fer de la machine M2 est nettement plus faible que celui de la machine M1 (20\% environ).

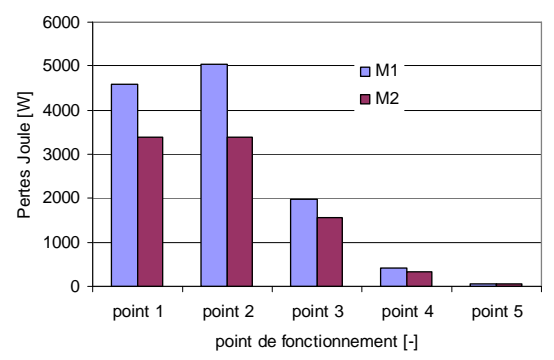

Figure 8. Les pertes Joule des deux machines sur différents points de fonctionnement.

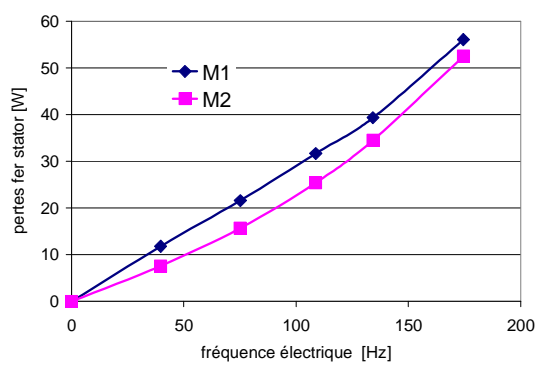

Figure 9.a. Évolution des pertes fer en fonction de la fréquence électrique pour les machines $M 1$ et M2.

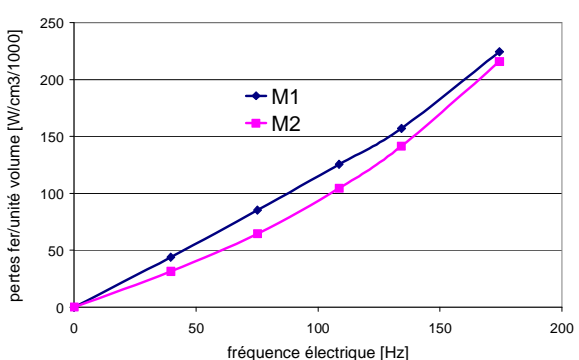

Figure 9.b. Évolution des pertes fer par unité de volume en fonction de la fréquence électrique pour les machines M1 et M2.

\subsection{Pertes par courants de Foucault dans les aimants permanents}

Nous évaluons maintenant les pertes par courants de Foucault dans les aimants permanents dans les deux machines M1 et M2. Ces pertes sont calculées numériquement (par la méthode des éléments finis 2D) pour les fonctionnements à vide et en charge des machines.

Les Tableaux 3 à 6 résument les résultats de simulations par éléments finis pour les pertes par courants de Foucault dans les aimants permanents des deux machines, 
Comparaison de deux types de bobinage pour la réalisation de moteur-roues à aimants permanents destinés à la propulsion d'un véhicule hybride urbain

ainsi que les pertes dans les aimants de la machine M2 (i.e., fractionnaire avec un bobinage à pas dentaire) avec segmentation des aimants en deux blocs selon la direction orthoradiale.

Tableau 3. Pertes par courants de Foucault dans les aimants permanents pour la machine M1 (i.e., non-fractionnaire avec un bobinage à pas diamétral).

\begin{tabular}{|l|llllll|}
\hline $\begin{array}{l}\text { Courant/ } \\
\text { Vitesse }\end{array}$ & 0A & $\mathbf{1 5} \mathbf{A}$ & $\mathbf{3 0} \mathbf{A}$ & $\mathbf{4 5} \mathbf{A}$ & $\mathbf{6 0} \mathbf{A}$ & $\mathbf{7 5} \mathbf{A}$ \\
\hline N2 & & $0,33 \mathrm{~W}$ & $0,8 \mathrm{~W}$ & $1,46 \mathrm{~W}$ & $2,431 \mathrm{~W}$ & $3,7 \mathrm{~W}$ \\
N3 & & $1,513 \mathrm{~W}$ & $2,754 \mathrm{~W}$ & $5,06 \mathrm{~W}$ & & \\
N4 & & $3,084 \mathrm{~W}$ & $5,78 \mathrm{~W}$ & & & \\
N5 & & $5,032 \mathrm{~W}$ & & & & \\
N6 & $7,38 \mathrm{~W}$ & & & & & \\
\hline
\end{tabular}

Tableau 4. Pertes par courants de Foucault dans les aimants permanents pour la machine M2 (i.e., fractionnaire avec un bobinage à pas dentaire).

\begin{tabular}{|l|llllll|}
\hline $\begin{array}{l}\text { Courant/ } \\
\text { Vitesse }\end{array}$ & 0A & 10A & 20 A & 30 A & 40 A & 50 A \\
\hline N2 & & $3,12 \mathrm{~W}$ & $5,56 \mathrm{~W}$ & $10,23 \mathrm{~W}$ & $16,23 \mathrm{~W}$ & $25,15 \mathrm{~W}$ \\
N3 & & $11,02 \mathrm{~W}$ & $19,41 \mathrm{~W}$ & $35,36 \mathrm{~W}$ & & \\
N4 & & $24,03 \mathrm{~W}$ & $40,41 \mathrm{~W}$ & & & \\
N5 & & $36,27 \mathrm{~W}$ & & & & \\
N6 & $53,6 \mathrm{~W}$ & & & & & \\
\hline
\end{tabular}

Tableau 5. Pertes par courants de Foucault dans les aimants permanents pour la machine M2 (i.e., fractionnaire avec un bobinage à pas dentaire) avec un découpage des aimants en 2 segments selon la direction orthoradiale.

\begin{tabular}{|l|llllll|}
\hline $\begin{array}{l}\text { Courant/ } \\
\text { Vitesse }\end{array}$ & 0A & 10A & 20 A & 30 A & 40 A & 50 A \\
\hline N2 & & $1,43 \mathrm{~W}$ & $2,26 \mathrm{~W}$ & $3,7 \mathrm{~W}$ & $5,52 \mathrm{~W}$ & $7,58 \mathrm{~W}$ \\
N3 & & $4,98 \mathrm{~W}$ & $7,95 \mathrm{~W}$ & $13,18 \mathrm{~W}$ & & \\
N4 & & $10,89 \mathrm{~W}$ & $16,85 \mathrm{~W}$ & & & \\
N5 & & $17,12 \mathrm{~W}$ & & & & \\
N6 & $26,52 \mathrm{~W}$ & & & & & \\
\hline
\end{tabular}

Les résultats montrent qu'il y a plus de pertes dans les aimants de la machine utilisant un bobinage dentaire. Ces pertes sont en effet dues aux sous-harmoniques $\mathrm{du}$ champ dans l'entrefer existants du fait du bobinage à pas fractionnaire (Magnussen, 2007). Ces harmoniques d'amplitudes élevées créent des champs 
tournants non synchrones à l'origine de courants de Foucault intenses dans les aimants. Cet inconvénient peut être limité par la segmentation des aimants dans le sens orthoradial (cf. Tableau 5) et/ou dans le sens axial. On notera que même si les pertes dans les aimants sont intrinsèquement un phénomène $3 \mathrm{D}$, du fait du rebouclage des courants de Foucault dans les aimants selon la direction orthoradiale, on suppose que les tendances des valeurs réelles sont les mêmes que celles observées sur les valeurs calculées avec un hypothèse 2D.

\section{Le défluxage et les inductances}

\subsection{Introduction au défluxage}

Un schéma électrique équivalent monophasé d'une MSAP à pôles lisses est représenté sur la Figure 10 où $E, R, L_{c y c l}$ et $I$ sont réciproquement l'amplitude de la force électromotrice, la résistance, l'inductance cyclique et l'amplitude efficace du courant d'alimentation d'une phase de la machine.

La tension d'alimentation de la machine produite par l'onduleur est limitée par la tension du bus continu. Lorsque la tension maximale est atteinte, l'augmentation de la vitesse peut être obtenue par injection d'un courant réactif qui s'oppose au flux constant créé par les aimants. On parle alors de défluxage (El-Refaie, 2006, Soong, 2002, Multon, 1995).

Le défluxage permet ainsi d'augmenter le nombre de spires du bobinage de la machine et de limiter le courant maximal absorbé par la machine pour produire le couple maximal, ce qui permet de diminuer la puissance dimensionnante de l'onduleur. Le diagramme de Fresnel de la Figure 11 illustre ce fonctionnement. Les équations électriques de la machine sont les suivantes :

- La tension globale d'alimentation de la machine est exprimée par (cf. Figure 10):

$\underline{V}=\underline{E}+R \cdot \underline{I}+j \cdot L_{c y c l} \cdot \omega \cdot \underline{I}$

- Les tensions sur l'axe d et l'axe q s'exprime par :

$V d=R \cdot I d+L_{c y c l} \cdot \omega \cdot I q$

$V q=E+R \cdot I q-L_{c y c l} \cdot \omega \cdot I d$

- L'amplitude de la tension de phase vaut (cf. Figure 11) :

$V=\sqrt{V d^{2}+V q^{2}}$ 


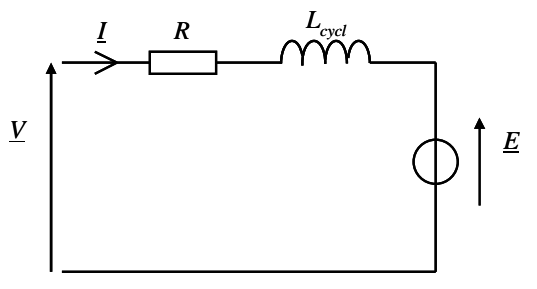

Figure 10. Schéma électrique équivalent monophasé d'une MSAP à pôles lisses.

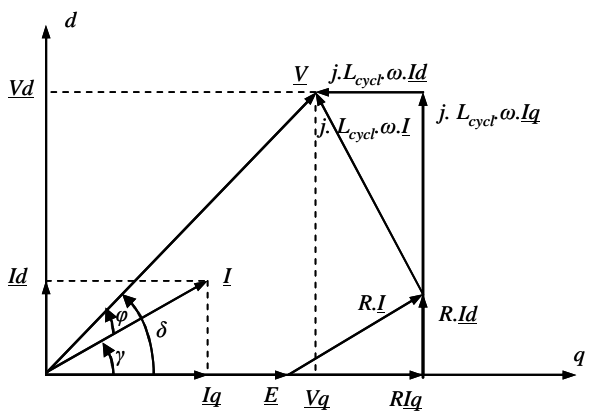

Figure 11. Diagramme vectoriel du schéma électrique d'une MSAP.

On notera aussi que $I q$ est la composante du courant nécessaire pour produire le couple électromagnétique du moteur. Nous remarquons aussi que dans les équations décrites ci-dessus la valeur de la tension de phase $V$ dépend fortement de la valeur de l'inductance cyclique $L_{c y c l}$ de la machine. C'est pour cette raison qu'il est important d'évaluer correctement cette inductance, et ce afin d'estimer la capacité de défluxage de la machine, c'est-à-dire la capacité à augmenter la vitesse de la machine à tension efficace d'alimentation constante. Dans le paragraphe suivant, nous évaluons donc les différentes valeurs des inductances des deux machines considérées. 


\subsection{Détermination les valeurs des inductances}

\subsubsection{Inductance propre et mutuelle de la machine $M 1$}

Le calcul de l'inductance propre et de la mutuelle d'une phase d'une machine à pas diamétral est classique et il a par exemple été rappelé dans (Espanet, 1999). L'inductance propre d'une phase de la machine à pas diamétral s'exprime par :

$$
L_{\text {prop_M1 }}=\sum_{\text {nimpair }}^{N_{h}} \mu_{0} \cdot n s^{2} \cdot \frac{4 \cdot p \cdot q}{n \cdot \pi} \cdot l f e r \cdot\left(\frac{\sin \left(n \cdot p \cdot \frac{\theta_{e}}{2}\right)}{n \cdot p \cdot \frac{\theta_{e}}{2}}\right)^{2} \cdot\left[\frac{\left(\frac{R r}{R s c}\right)^{n \cdot p}+\left(\frac{R s c}{R r}\right)^{n \cdot p}}{\left(\frac{R r}{R s c}\right)^{n \cdot p}-\left(\frac{R s c}{R r}\right)^{n \cdot p}}\right]
$$

où $R r, R s c$ sont réciproquement le rayon du rotor et le rayon d'alésage de la machine corrigé par le coefficient de Carter (Espanet 1999), $\theta_{e}$ est l'ouverture d'une encoche en radian, et $n$ est le rang harmonique de la série.

La mutuelle entre deux phases de la machine s'exprime quant à elle par :

$$
\begin{aligned}
& M_{\text {mutuelle_M1 }}=\sum_{\text {nimpair }}^{N_{h}}-\mu_{0} \cdot n s^{2} \cdot \frac{4 \cdot p \cdot q}{n \cdot \pi} \cdot \text { lfer } \cdot\left(\frac{\sin \left(n \cdot p \cdot \frac{\theta_{e}}{2}\right)}{n \cdot p \cdot \frac{\theta_{e}}{2}}\right)^{2} \cdot\left[\frac{\left(\frac{R r}{R s c}\right)^{n \cdot p}+\left(\frac{R s c}{R r}\right)^{n \cdot p}}{\left(\frac{R r}{R s c}\right)^{n \cdot p}-\left(\frac{R s c}{R r}\right)^{n \cdot p}}\right] \cdots \\
& \cdot \sin \left(n \cdot \frac{\pi}{2}\right) \cdot \sin \left(n \cdot \frac{\pi}{6}\right)
\end{aligned}
$$

\subsubsection{Inductance propre et mutuelle de la machine $M 2$}

Nous proposons ensuite d'évaluer les valeurs de l'inductance propre et de la mutuelle d'une machine à pas dentaire. Pour cela, nous utilisons une approche énergétique présentée dans (Zhu, 1995). L'inductance propre d'une phase est calculée par la relation énergétique de l'équation 18 où $W_{p 1_{-} M_{2}}$ représente l'énergie magnétique stockée dans la machine lorsque seule la phase 1 est alimentée $\left(I_{l}=I\right.$ et $I_{2}=I_{3}=0$ ).

$$
W_{p 1 \_M 2}=\frac{1}{2} \cdot L_{\text {prop } \_22} \cdot I_{1}^{2}
$$

En supposant la perméabilité du fer infinie, le calcul de cette énergie est obtenu en faisant l'intégration du produit du potentiel vecteur $A_{r I}$ créé par le seul courant de la phase 1 et de la densité surfacique (correspondant au même courant) $j s$ sur la surface de l'entrefer Sent :

$$
W_{p 1 \_M 2}=\frac{1}{2} \cdot \iint_{\text {Sent }} A_{z}^{I}\left(R s c, \theta_{s}\right) \cdot j s\left(\theta_{s}\right) \cdot d \operatorname{Sent}
$$


Comparaison de deux types de bobinage pour la réalisation de moteur-roues à aimants permanents destinés à la propulsion d'un véhicule hybride urbain

où l'expression du potentiel vecteur est donnée en Annexe 2.

Après calcul de l'intégrale de l'équation 19, l'expression de l'inductance propre peut être obtenue :

$$
\begin{aligned}
& L_{\text {prop_M } 2}=\frac{l \text { fer }}{2} \cdot \mu_{0} \cdot \frac{\pi}{\left(\frac{\theta_{o e}}{2}\right)^{2}} \cdot \ldots \\
& \cdot \sum_{\text {nimpair }}^{n_{h}} \frac{1}{n} \cdot \frac{M_{3}(n)+M_{4}(n, R s c)}{M_{2}(n, R s c)-M_{1}(n, R s c)} \cdot\left[D_{s w s}\left(n, \zeta_{o e}, \theta_{o d}\right)^{2}+D_{s w c}\left(n, \zeta_{o e}, \theta_{o d}\right)^{2}\right]
\end{aligned}
$$

où $D_{s w s}$ et $D_{s w c}$ sont les coefficients de la distribution spatiale du bobinage dentaire, et $M_{i}(i=1 . .4)$ sont des paramètres intervenant dans l'expression du potentiel vecteur créé par le courant alimentation de la phase 1 seule (voir Annexe 2).

La mutuelle entre deux phases de la machine à pas dentaire est également calculée de la même façon par l'intermédiaire d'une approche énergétique. On considère que les phases 1 et 2 sont alimentées, respectivement par les courants $I_{l}$ et $I_{2}$ et on calcule l'énergie magnétique totale emmagasinée dans la machine :

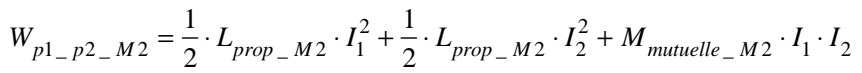

Le troisième terme de l'équation précédente permet finalement d'obtenir l'expression de la mutuelle entre la phase 1 et la phase 2 de la machine M2 :

$$
\begin{aligned}
& M_{\text {mutuelle_M2 }}=\frac{l \text { fer }}{2} \cdot \mu_{0} \cdot \frac{1}{\left(\frac{\theta_{o e}}{2}\right)^{2}} \cdot \ldots \\
& \cdot \sum_{\text {nimpair }}^{n_{h}} \frac{1}{n} \cdot \frac{M_{3}(n)+M_{4}(n, R s c)}{M_{2}(n, R s c)-M_{1}(n, R s c)} \cdot \pi \cdot\left[\begin{array}{l}
D_{s w s}\left(n, \zeta_{o e}, \theta_{o d}\right)^{2}+\ldots \\
+D_{s w c}\left(n, \zeta_{o e}, \theta_{o d}\right)^{2}
\end{array}\right] \cdot \cos \left[n \cdot \frac{2 \cdot \pi}{N_{p h}}\right]
\end{aligned}
$$

où $N_{p h}$ représente le nombre de phases dans la machine (dans notre cas $N_{p h}=3$ ).

En outre, dans le cas de la machine M2, où le bobinage est dentaire avec deux bobines par encoche, il existe également un couplage mutuel au sein des encoches entre deux bobines d'une même encoche, mais situé à l'extrémité de deux phases différentes. Cette mutuelle est calculée dans les paragraphes suivants concernant les inductances de fuites.

\subsubsection{Inductance de fuites de la machine M1}

Les inductances de fuites proviennent des flux de fuites dans la machine (Foggia, 1999). Nous pouvons distinguer les fuites dans les encoches, les fuites différentielles, les fuites au niveau des têtes des bobines, etc. Ici nous allons considérer uniquement l'inductance de fuites dans deux parties. La première 
correspond au flux traversant l'encoche et la seconde correspond au flux traversant les isthmes entre les becs de dents (Foggia, 1999). Nous ignorons volontairement les fuites au niveau des têtes de bobine, ce choix pouvant être particulièrement discutable pour les machines considérées dans cette étude qui ont un diamètre relativement important par rapport à la longueur.

L'inductance de fuites d'une machine à pas diamétral se calcule par :

$$
L_{\text {fute_M1 }}=\mu_{0} \cdot 2 p \cdot q \cdot n s^{2} \cdot \text { lfer } \cdot\left(\frac{2 \cdot h_{1}}{3 \cdot\left(b_{1}+b_{2}\right)}+\frac{b_{3}}{b_{2}-b_{4}} \cdot \ln \left(\frac{b_{2}}{b_{4}}\right)+\frac{h_{4}-h_{2}}{b_{4}}\right)
$$

où $h_{i}, b_{i}(i=1 . .4)$ sont les paramètres géométriques caractéristiques d'une encoche (cf. Figure 12).

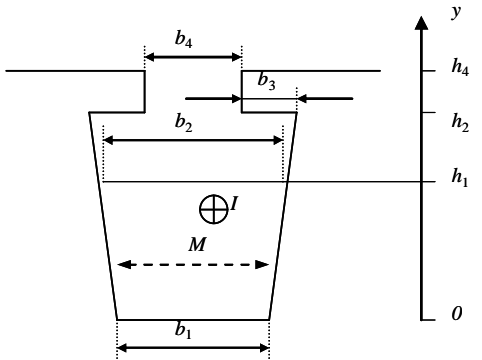

Figure 12. Paramètres géométriques caractéristiques d'une encoche quelconque.

\subsubsection{Inductance de fuites de la machine M2}

Le calcul de l'inductance de fuites dans une encoche de la machine M2 est moins classique car une encoche est occupée par deux bobines. Par ailleurs, lorsque ces deux bobines n'appartiennent par à la même phase, il existe également un couplage mutuel entre les deux bobines. Pour illustrer ce phénomène, la figure 13 montre les lignes de champ dans une telle encoche. On distingue deux bobines, seule celle de droite est alimentée et on distingue deux types de lignes de champ :

- les lignes qui se referment dans les deux bobines et qui correspondent à un effet de mutuelle inductance ; on pourrait parler de mutuelle d'encoche ;

- les lignes qui se referment dans uniquement dans la bobine alimentée et qui correspondent à un effet d'inductance propre.

L'inductance de fuites élémentaire correspondant à une demi-bobine contenue dans une encoche s'exprime par (Zhu, 1995) : 
Comparaison de deux types de bobinage pour la réalisation de moteur-roues à aimants permanents destinés à la propulsion d'un véhicule hybride urbain

$$
L_{\text {fuite_elem_M2 }}=\mu_{0} \cdot n s^{2} \cdot \operatorname{lfer} \cdot\left(\frac{2 \cdot h_{1}}{3 \cdot\left(b_{1}+b_{2}\right)}+\frac{b_{3}}{b_{2}-b_{4}} \cdot \ln \left(\frac{b_{2}}{b_{4}}\right)+\frac{h_{4}-h_{2}}{b_{4}}\right)
$$

Et, dans le cas de la machine M2, l'inductance de fuites totale vaut alors :

$$
L_{\text {fuite_M2 }}=2 \cdot N_{b m} \cdot L_{\text {fuite_elem_M } M}-2 \cdot\left(\frac{N_{b m}}{2}-1\right) \cdot 2 \cdot M_{\text {fuite_elem_M } 2}
$$

où $N_{b m}$ est le nombre de bobines de la machine M2.

De plus, la mutuelle élémentaire entre deux bobines d'une même encoche est calculée par (Zhu, 1995):

$$
M_{\text {fuite_elem_M2 }}=\frac{-1}{2} \cdot \mu_{0} \cdot n s^{2} \cdot \text { lfer } \cdot\left(\begin{array}{l}
\frac{h_{4}-h_{2}}{b_{4}}+\frac{2 \cdot h_{1}}{3 \cdot\left(b_{1}+b_{2}\right)}-\frac{\frac{\left(b_{1}+b_{2}\right)}{2}}{12 \cdot h_{2}}+ \\
\sum_{1}^{100} \frac{1}{n \cdot \pi} \cdot \frac{1+e^{\frac{-8 n \cdot \pi \cdot h_{2}}{b_{1}+b_{2}}}}{1-e^{\frac{-8 n \cdot \pi \cdot h_{2}}{b_{1}+b_{2}}}} \cdot\left[\frac{\sin \left[\frac{2 \cdot n \cdot \pi \cdot b_{4}}{b_{1}+b_{2}}\right]}{\frac{2 \cdot n \cdot \pi \cdot b_{4}}{b_{1}+b_{2}}}\right]
\end{array}\right)
$$

Il convient ainsi d'ajouter à l'expression de la mutuelle donnée à l'équation 22 , la mutuelle existant entre deux bobines dans une même encoche à l'extrémité de deux phases différentes. Ce couplage mutuel existe deux fois dans le cas de la machine M2, donc la mutuelle totale de la machine M2 se calcule par :

$$
M_{\text {mut_tot }} M 2=M_{\text {mutuelle }} M 2+2 \cdot M_{\text {fuite }} \text { elem }_{-} M 2
$$

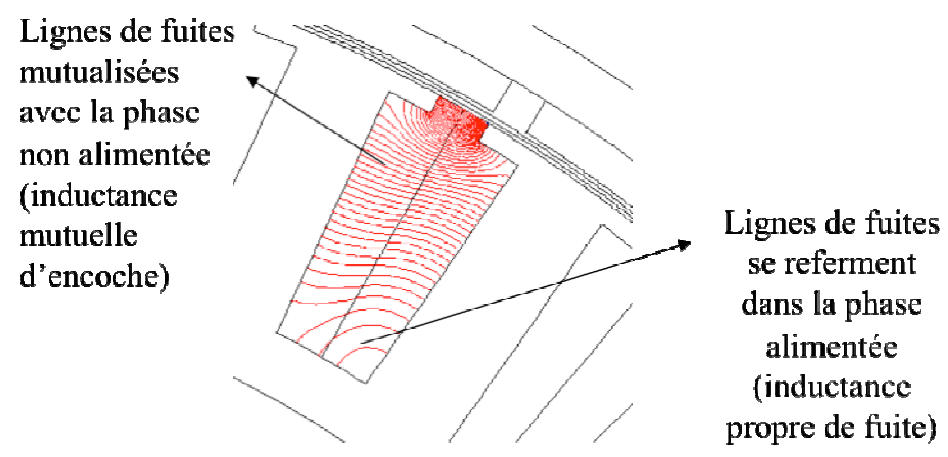

Figure 13. Lignes de fuites dans une encoche ayant deux bobines appartenant à deux phases différentes de la machine M2 (seule la bobine de droite est alimentée) 


\subsubsection{Inductances cycliques}

Les valeurs des inductances cycliques des deux machines M1 et M2 sont déduites grâce aux expressions des inductances et mutuelles calculées dans les paragraphes précédents :

$$
L_{\text {cycl }}=L_{\text {prop }}-M_{\text {mutuelle }}+L_{\text {fuite }}
$$

Les valeurs des inductances cycliques d'une phase des deux machines sont également comparées à celles obtenues par la méthode des éléments finis 2D (cf. Tableaux 6 et 7). Nous trouvons des valeurs concordantes entre le modèle analytique et le modèle numérique.

Tableau 6. Les valeurs des inductances de la machine M1.

\begin{tabular}{lccl} 
& \multicolumn{4}{c}{ Pas diamétral } \\
\hline & Ana & EF & {$[\%]$} \\
& 0,32 & 0,637 & \\
Inductance propre, $L_{\text {prop }}[\mathrm{mH}]$ & 0,397 & & \\
Inductance de fuites, $L_{\text {fuite }}[\mathrm{mH}]$ & $-0,103$ & $-0,107$ & \\
Mutuelle, $M_{\text {mut }}[\mathrm{mH}]$ & 0,821 & 0,744 & 10,34 \\
$\begin{array}{l}\text { Inductance cyclique, } L_{\text {cycl }}[\mathrm{mH}] \\
\text { Nombre de bobines en série dans une } \\
\text { phase complète de la machine }[-]\end{array}$ & 170 & 170 & \\
$\begin{array}{l}\text { Inductance cyclique pour une seule } \\
\text { bobine en série dans une phase }[\mathrm{nH}]\end{array}$ & & & \\
\end{tabular}

Tableau 7. Les valeurs des inductances de la machine M2.

Pas dentaire

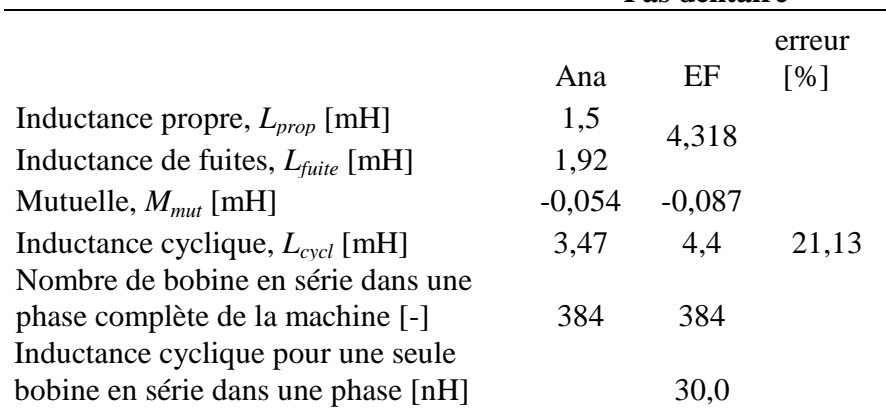


Comparaison de deux types de bobinage pour la réalisation de moteur-roues à aimants permanents destinés à la propulsion d'un véhicule hybride urbain

Nous remarquons que pour la machine M2, la mutuelle est très faible devant l'inductance propre, ce qui est cohérent avec la configuration du bobinage de cette machine présentée Figure 3. En effet, dans la machine à bobinage dentaire, les effets mutuels ont lieu aux extrémités des groupes de bobines, alors que dans un bobinage à pas diamétral les bobines sont entrelacées et approximativement la totalité du flux d'une phase est mutualisé avec les deux autres phases (la première moitié avec une deuxième phase et la deuxième moitié avec la troisième phase).

Nous remarquons également que la valeur de l'inductance cyclique de la machine M2 (machine fractionnaire avec un bobinage à pas dentaire) est nettement supérieure à celle de la machine M1 (machine non fractionnaire avec un bobinage à pas diamétral). La raison principale provient dans ce cas particulier de la différence du nombre total de spires en série dans une phase (cf. Tableaux 6 et 7). En effet, le dimensionnement optimal des deux machines n'a pas été fait avec les mêmes contraintes sur le défluxage. La machine M1 étant alimentée en créneaux, il a été choisi de pouvoir atteindre la pleine vitesse sans déphasage du courant, pour ne pas provoquer trop d'ondulations de couple, alors que la machine M2 a été optimisée en laissant une possibilité de défluxage. De ce fait, le nombre total de spires en série dans une phase est plus grand dans le cas de la machine M2 utilisant un bobinage à pas dentaire. Pour aller plus loin dans la comparaison, nous avons calculé dans les Tableaux 6 et 7 les inductances cycliques pour une seule spire de bobinage en considérant uniquement le calcul numérique par éléments finis 2D (on suppose que c'est le calcul le plus précis). Nous constatons que l'inductance par spire est plus élevée pour la machine à pas dentaire. La cause provient principalement des fuites d'encoches qui sont plus importantes pour la machine utilisant un bobinage à pas dentaire. En effet le calcul (par le modèle analytique) de l'inductance propre d'entrefer pour une spire donne deux valeurs relativement proches : $10 \mathrm{nH}$ pour la machine M1 et $11 \mathrm{nH}$ pour la machine M2. De plus, rappelons que l'effet d'inductance mutuelle est très limité pour la machine utilisant un bobinage à pas dentaire. Il en résulte nécessairement que les $20 \%$ d'écart entre les inductances cycliques des machines M1 et M2 (au profit de M2) sont liés en grande partie aux fuites.

Notons toutefois que pour la machine M1 à bobinage à pas diamétral, l'alimentation par des créneaux de courant ne permet pas de déphaser fortement les courants au risque de provoquer d'importantes ondulations de couple; une commutation vers une alimentation sinusoïdale à une vitesse intermédiaire peut éventuellement être une solution pour défluxer la machine sans introduire de trop fortes ondulations de couple et ainsi réduire les contraintes de dimensionnement de l'onduleur.

$$
\begin{aligned}
& \text { Dans les tableaux } 6 \text { et } 7, \text { l'erreur de calcul est calculée par : } \\
& \text { Err }=\frac{\left|L_{c y c l_{-} A N A}-L_{c y c l_{-} E F}\right|}{L_{c y c l_{-} E F}} \cdot 100
\end{aligned}
$$




\section{Forces magnétiques et problème de vibration des machines}

Les Figures 14 et 15 représentent les vecteurs forces auxquels sont soumis une dent dans le cas des deux machines considérées dans cette étude. Les Figures 16 à 19 représentent quant à elles les composantes radiales des forces magnétiques qui s'exercent sur les dents d'une paire de pôles de la machine M1 utilisant un bobinage à pas diamétral et sur la moitié des dents de la machine M2 utilisant un bobinage à pas dentaire. Les forces correspondant à la machine à pas diamétral sont tracées pour une seule position du rotor par rapport au stator, alors que les forces correspondant à la machine à pas dentaire sont tracées pour trois positions du rotor par rapport au stator $\left(0^{\circ}, 4,2^{\circ}\right.$ et $\left.8,4^{\circ}\right)$. Les forces sont calculées au point de fonctionnement 2 où le couple important nécessite des courants de phase et donc un champ statoriques intense.

Il apparaît clairement que les forces sont plus importantes dans le cas de la machine utilisant un bobinage à pas dentaire. Cela s'explique par le fait que les dents sont nettement moins nombreuses dans le cas de la machine M2 (36 au lieu de 102) pour un même nombre d'aimants; les dents de la machine M2 sont alors plus large et la résultante des efforts magnétiques sur la dent est plus intense. On observe également que la machine à pas dentaire présente de plus importantes variations des forces magnétiques s'exerçant sur les dents du stator. Ces variations, liées aux harmoniques du champ magnétique créées par le bobinage, peuvent être à l'origine de vibrations dans la machine. Il convient alors de bien calculer les fréquences des harmoniques et de vérifier que ces fréquences ne risquent pas d'exciter les structures mécaniques du stator ou du rotor au voisinage d'une fréquence de résonance, ou de renforcer ces structures en vue de limiter les risques de ruptures mécaniques.

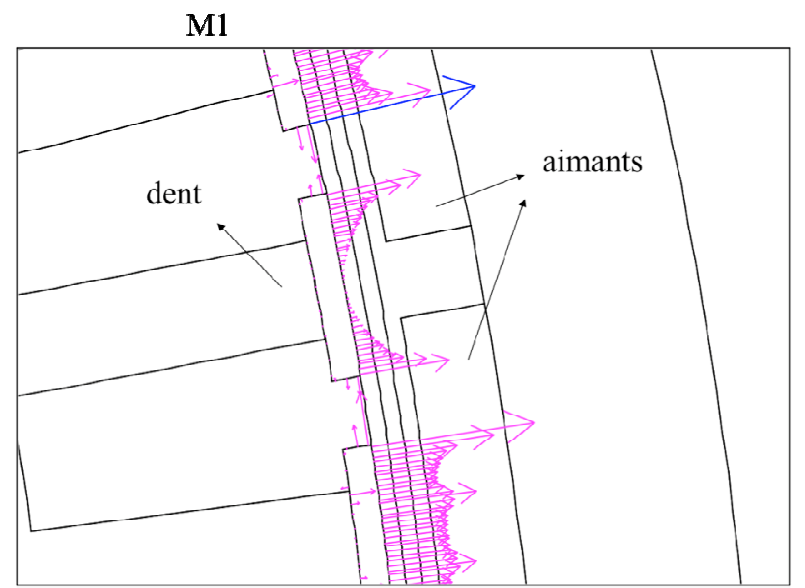

Figure 14. Vecteurs forces sur une dent de la machine M1 au point de fonctionnement 2. 
Comparaison de deux types de bobinage pour la réalisation de moteur-roues à aimants permanents destinés à la propulsion d'un véhicule hybride urbain

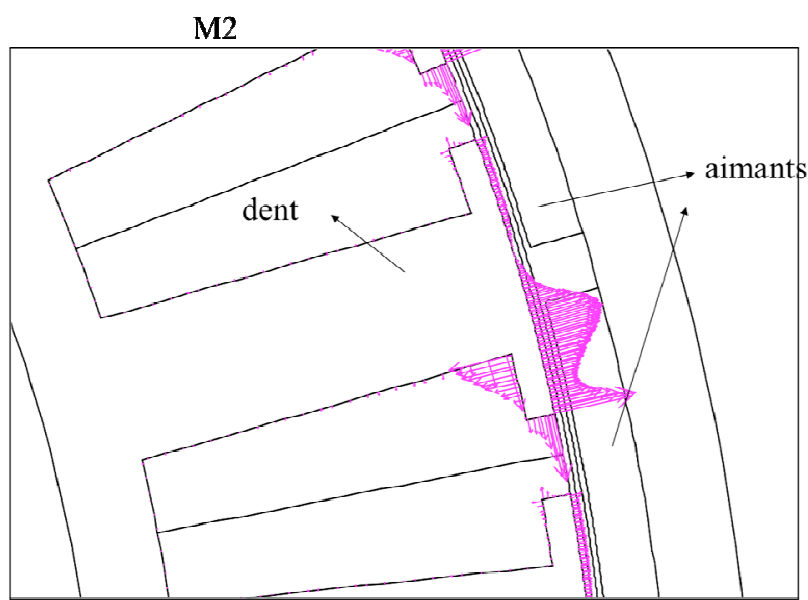

Figure 15. Vecteurs forces sur une dent de la machine M2 au point de fonctionnement 2 .

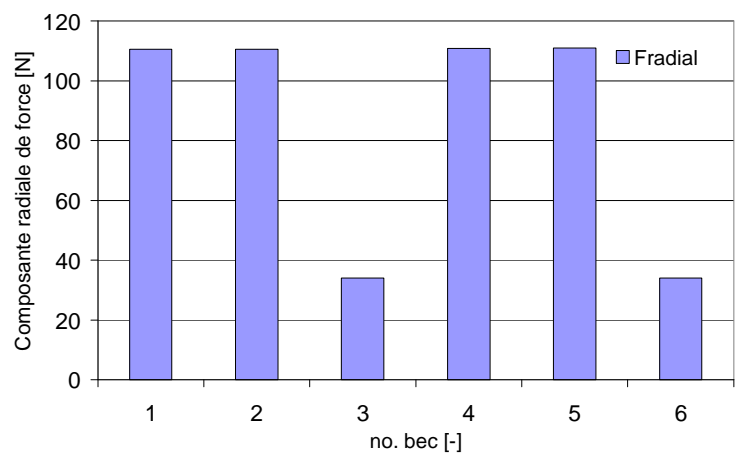

Figure 16. Composantes radiales des forces magnétiques s'exerçant sur les becs des 6 dents d'une paire de pôles de la machine M1 au point de fonctionnement 2. 


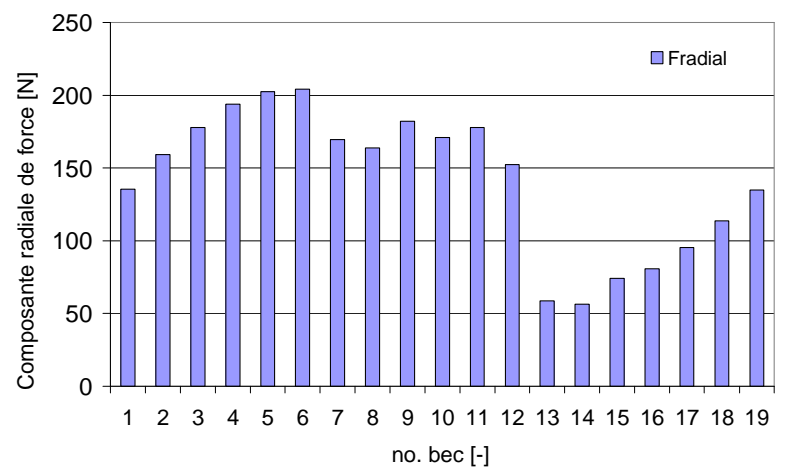

Figure 17. Composantes radiales des forces magnétiques s'exerçant sur les becs des 19 dents de la machine M2 au point de fonctionnement 2 (position du rotor par rapport au stator valant $0^{\circ}$ ).

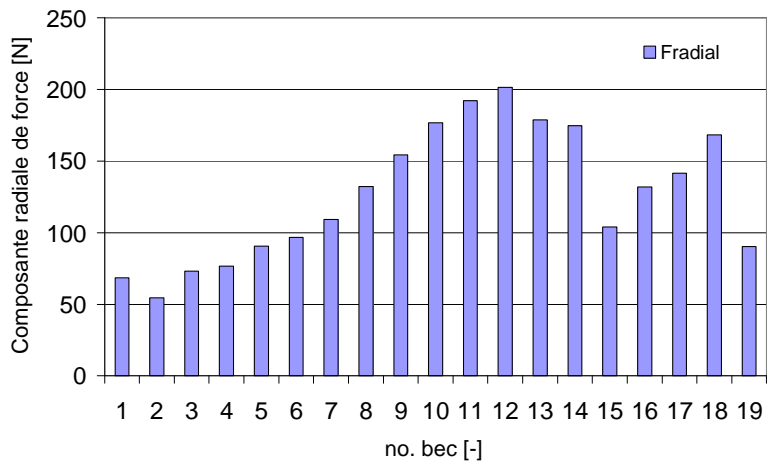

Figure 18. Composantes radiales des forces magnétiques s'exerçant sur les becs des 19 dents de la machine M2 au point de fonctionnement 2 (position du rotor par rapport au stator valant $4,2^{\circ}$ ). 
Comparaison de deux types de bobinage pour la réalisation de moteur-roues à aimants permanents destinés à la propulsion d'un véhicule hybride urbain

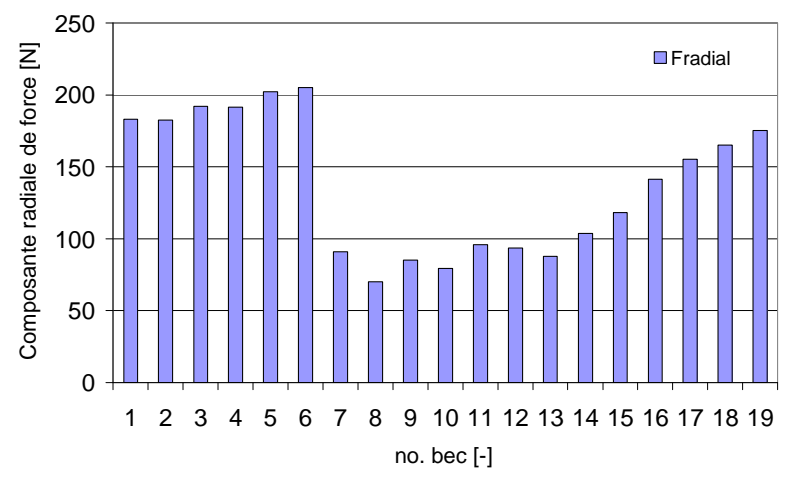

Figure 19. Composantes radiales des forces magnétiques s'exerçant sur les becs des 19 dents de la machine M2 à pas dentaire au point de fonctionnement 2 (position du rotor par rapport au stator valant $8,4^{\circ}$ ).

\section{Conclusion}

Une comparaison de deux machines synchrones à aimants permanents qui diffèrent par le nombre d'encoches par pôle et par phase et par le type de bobinage de machine a été présentée dans cet article. La machine est destinée à la réalisation d'un moteur-roue pour la propulsion électrique d'un véhicule hybride de petite puissance pour un usage urbain. La première machine est à nombre d'encoches par pôle et par phase entier et utilise un bobinage à pas diamétral. La seconde machine est à nombre d'encoches par pôle et par phase fractionnaire (non entier) et vérifie la relation $N d=2 . p \pm 2$; le bobinage est concentrique autour des dents et à double couche (toutes les dents sont bobinées). Le calcul de plusieurs grandeurs électromagnétiques a été abordé dans l'article. Au-delà des remarques liées au cas particulier traité dans cet article, les auteurs on pu dégager des conclusions dont la justification permet d'augmenter la généralité de portée.

Les principales conclusions sont ainsi les suivantes :

- La machine à pas dentaire a une force électromotrice quasi-sinusoïdale et elle présente un très faible couple de détente, ce qui sont des avantages par rapport à la machine à pas diamétral.

- La machine à bobinage dentaire est plus compacte car ses têtes de bobines sont plus courtes. Cela permet globalement de réduire les pertes par effet Joule de la machine à bobinage dentaire par rapport à la machine à bobinage réparti. Ceci étant, l'optimisation de la machine sur la base de 
différents critères peut conduire à des résultats en contradiction avec cette remarque générale.

- L'étude a également montré que la machine à bobinage dentaire possède des pertes fer plus faibles du fait de la réduction du volume de fer.

- L'étude a montré aussi que la machine à bobinage dentaire a une plus grande valeur d'inductance cyclique (principalement du fait de fuites plus importantes si on compare les inductances pour une seule spire de bobinage), ce qui facilite le défluxage de la machine et le dimensionnement de l'onduleur. Cela peut toutefois se payer au niveau du facteur de puissance et donc du dimensionnement du condensateur de filtrage du bus continu en entrée de l'onduleur.

- Par ailleurs, l'étude a mis en évidence quelques inconvénients de la machine à bobinage dentaire. Elle présente plus de pertes dans les aimants, et elle présente des variations des forces magnétiques sur les dents du stator plus importantes (pouvant être à l'origine de vibrations et de bruit). Ces effets sont dus aux harmoniques du champ magnétique créés par le bobinage particulier de la machine. Pour réduire les pertes, les auteurs proposent de segmenter les aimants. L'étude vibro-acoustique fera l'objet d'une prochaine étude, sachant que deux options sont envisageables en cas de bruit ou vibrations : la modification du nombre de dents et de pôles (ce qui n'est pas très souhaitable car cela risque de modifier les performances en terme de couple spécifique et/ou de rendement Joule) ou la modification mécanique du stator et du rotor pour repousser les fréquences de résonnance du moteur.

\section{Remerciements}

Ce travail a été réalisé dans le cadre du projet PHEBUS de l'édition 2008 du programme PREDIT Véhicules Propres et Economes. Il est financé par l'Agence Nationale de la Recherche (ANR) et soutenu par l'Agence de l'Environnement et de la Maîtrise de l'Energie (ADEME). 
Comparaison de deux types de bobinage pour la réalisation de moteur-roues à aimants permanents destinés à la propulsion d'un véhicule hybride urbain

\section{Annexe}

\subsection{Coefficients de la distribution spatiale du bobinage dentaire}

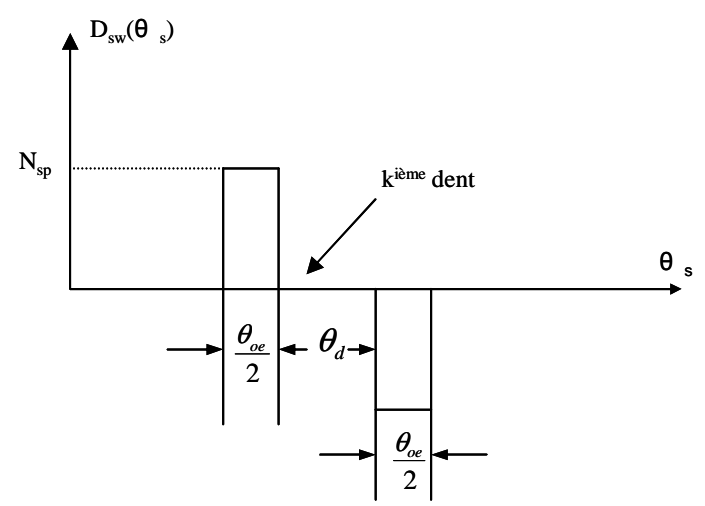

Figure 20. Représentation graphique de la $k^{\text {ième }}$ bobine dans la distribution du bobinage dentaire.

L'expression analytique de la distribution spatiale du bobinage dentaire a été développée en série Fourier (cf. Figure 20). On calcule tout d'abord le coefficient d'ouverture d'encoche statorique $K_{o e}$ et le coefficient d'ouverture des bobines statoriques $K_{o b}$; ce sont des fonctions de $\zeta_{o e}$, l'ouverture d'une encoche relativement au pas dentaire, et de $\theta_{o d}$, le pas dentaire au stator :

$$
\begin{aligned}
K_{o e}\left(n, \zeta_{o e}, \theta_{o d}\right) & =\frac{\sin \left(n \cdot \frac{\zeta_{o e} \cdot \theta_{o d}}{4}\right)}{n \cdot \frac{\zeta_{o e} \cdot \theta_{o d}}{4}} \\
K_{o b}\left(n, \zeta_{o e}, \theta_{o d}\right) & =\frac{\sin \left(n \cdot \frac{\left(\zeta_{o e}-2\right) \cdot \theta_{o d}}{4}\right)}{\left(n \cdot \frac{\left(\zeta_{o e}-2\right) \cdot \theta_{o d}}{4}\right)}
\end{aligned}
$$

La série de Fourier de la distribution spatiale du bobinage contient des termes en cosinus et des termes en sinus :

$$
\begin{gathered}
K d_{s}\left(n, \theta_{o d}\right)=\sum_{b=1}^{\frac{N_{b m}}{2}}\left[(-1)^{b+1} \cdot n \cdot \cos \left[n \cdot\left[(2 \cdot b-1) \cdot \frac{\theta_{o d}}{2}\right]\right]\right] \\
K d_{c}\left(n, \theta_{o d}\right)=\sum_{b=1}^{\frac{N_{b m}}{2}}\left[(-1)^{b+1} \cdot n \cdot \sin \left[n \cdot\left[(2 \cdot b-1) \cdot \frac{\theta_{o d}}{2}\right]\right]\right]
\end{gathered}
$$

où $N_{b m}$ est le nombre de bobines en série dans une phase de bobinage statorique. 
Les coefficients de distribution du bobinage, $D_{s w s}$ et $D_{s w c}$, sont alors exprimés en fonction des coefficients $K_{o e}, K_{o b}, K d_{s}$ et $K d_{c}$ comme suit :

$$
\begin{aligned}
& D_{s w s}\left(n, \zeta_{o e}, \theta_{o d}\right)=\frac{N_{s p}}{\pi} \cdot 2 \cdot K_{o e}\left(n, \zeta_{o e}, \theta_{o d}\right) \cdot K_{o b}\left(n, \zeta_{o e}, \theta_{o d}\right) \cdot K d_{s}\left(n, \theta_{o d}\right) \cdot \ldots \\
& \zeta_{o e} \cdot \theta_{o d} \cdot \frac{\left(\zeta_{o e}-2\right) \cdot \theta_{o d}}{4}
\end{aligned}
$$

et

$$
\begin{aligned}
& D_{s w c}\left(n, \zeta_{o e}, \theta_{o d}\right)=\frac{-N_{s p}}{\pi} \cdot 2 \cdot K_{o e}\left(n, \zeta_{o e}, \theta_{o d}\right) \cdot K_{o b}\left(n, \zeta_{o e}, \theta_{o d}\right) \cdot K d_{c}\left(n, \theta_{o d}\right) \cdot \ldots \\
& \cdot \zeta_{o e} \cdot \theta_{o d} \cdot \frac{\left(\zeta_{o e}-2\right) \cdot \theta_{o d}}{4}
\end{aligned}
$$

où $N_{s p}$ est le nombre de spires en série d'une bobine dans une encoche.

Finalement, la série de Fourier de la fonction de distribution spatiale du bobinage s'écrit :

$$
D_{s w}\left(\theta_{s}\right)=\sum_{n i m p a i r}^{N_{h}}\left\{D_{s w c}\left(n, \zeta_{o e}, \theta_{o d}\right) \cdot \cos \left[n \cdot\left(\theta_{s}-g \cdot \frac{2 \cdot \pi}{N_{p h}}\right)\right]+D_{s w s}\left(n, \zeta_{o e}, \theta_{o d}\right) \cdot \sin \left[n \cdot\left(\theta_{s}-g \cdot \frac{2 \cdot \pi}{N_{p h}}\right)\right]\right\}
$$

où $g(g=1,2,3)$ représente le numéro de la phase.

\subsection{Potentiel vecteur dans l'entrefer et les aimants produit par une seule phase dans le cas de la machine à bobinage dentaire.}

Pour obtenir l'expression du potentiel vecteur produit par le courant d'alimentation, les équations de Maxwell sont résolus dans deux régions: la première correspond à l'entrefer lisse corrigé par le coefficient de Carter et la seconde correspond aux aimants. Les coefficients d'intégration intervenant dans les expressions des potentiels vecteurs sont obtenus grâce aux conditions aux limites sur les deux régions. Après calculs, l'expression analytique du potentiel vecteur dans l'entrefer produit par le courant de la phase 1 seule est alors :

$$
A_{z}^{I}\left(r, \theta_{s}\right)=\sum_{\text {nimpair }}^{N_{h}} \mu_{0} \cdot \frac{\left[\begin{array}{l}
D_{s w s}\left(n, \zeta_{o e}, \theta_{o d}\right) \cdot \sin \left(n \cdot \theta_{s}\right)+\ldots \\
+D_{s w c}\left(n, \zeta_{o e}, \theta_{o d}\right) \cdot \cos \left(n \cdot \theta_{s}\right)
\end{array}\right] \cdot i_{1}(t)}{\frac{\theta_{o e}}{2}} \cdot \frac{1}{n} \cdot \frac{M_{3}(n)+M_{4}(n, r)}{M_{2}(n, r)-M_{1}(n, r)}
$$

où $\theta_{s}$ est l'angle dans un repère statorique.

Et l'expression du potentiel vecteur produit par le courant de la phase 1 seule dans la région des aimants est : 
Comparaison de deux types de bobinage pour la réalisation de moteur-roues à aimants permanents destinés à la propulsion d'un véhicule hybride urbain

$$
A_{z}^{I I}\left(r, \theta_{s}\right)=\sum_{\text {nimpair }}^{N_{h}} \mu_{0} \cdot \frac{\left[\begin{array}{l}
D_{s w s}\left(n, \zeta_{o e}, \theta_{o d}\right) \cdot \sin \left(n \cdot \theta_{s}\right)+\ldots \\
+D_{s w c}\left(n, \zeta_{o e}, \theta_{o d}\right) \cdot \cos \left(n \cdot \theta_{s}\right)
\end{array}\right] \cdot i_{1}(t)}{\frac{\theta_{o e}}{2}} \cdot \frac{1}{n} \cdot \frac{1}{M_{2}(n, r)-M_{1}(n, r)}
$$

où les expressions des $M_{i}(i=1 \ldots 4)$ sont :

$$
\begin{aligned}
& M_{1}(n, r)=\left(\frac{R s c}{r}\right)^{n} \cdot \frac{\left[\left(1+\frac{1}{\mu r_{a}}\right)+\left(1-\frac{1}{\mu r_{a}}\right) \cdot\left(\frac{R r}{R a}\right)^{2 \cdot n}\right]}{2} \\
& M_{2}(n, r)=\frac{\left[\left(1-\frac{1}{\mu r_{a}}\right) \cdot\left(\frac{R a}{R s c}\right)^{n} \cdot\left(\frac{R a}{r}\right)^{n}+\left(1+\frac{1}{\mu r_{a}}\right) \cdot\left(\frac{R r}{R s c}\right)^{n} \cdot\left(\frac{R r}{r}\right)^{n}\right]}{2} \\
& M_{3}(n)=\frac{\left(1+\frac{1}{\mu r_{a}}\right)+\left(1-\frac{1}{\mu r_{a}}\right) \cdot\left(\frac{R r}{R a}\right)^{2 \cdot n}}{2} \\
& M_{4}(n, r)=\frac{\left(1-\frac{1}{\mu r_{a}}\right) \cdot\left(\frac{R a}{r}\right)^{2 \cdot n}+\left(1+\frac{1}{\mu r_{a}}\right) \cdot\left(\frac{R r}{r}\right)^{2 \cdot n}}{2}
\end{aligned}
$$

où $\mu r_{a}$ est la perméabilité des aimants permanents, $R r$ et $R a$ sont respectivement le rayon du rotor et le rayon des aimants permanents.

Ces expressions du potentiel permettent finalement le calcul de l'énergie magnétique stockée dans la machine.

\section{Bibliographie}

Bianchi N., Bolonagni S., Dai Pré M. Grezzani G., «Design Considerations for FractionalSlot Winding Configurations of Synchronous Machines», IEEE Trans. on Energy Conversion, Vol. 42, No. 4, August 2006.

Chan C. C., Jiang J. Z., Chen G. H., Wang X. Y., and Chau K. T., «A Novel Polyphase Multipole Square-Wave Permanent Magnet Motor Drive for Electric Vehicles», IEEE Trans. On Industry Applications, Vol. 30, No. 5, Sep./Oct. 1994.

Cros J., Viarouge P., «Synthesis of High Performance PM Motors With Concentrated Windings», IEEE Trans. on Energy Conversion, Vol. 17, No. 2, June 2002.

El-Refaie A. M., Zhu Z. Q., Jahns T.M., Howe D., «Winding Inductances of Fractional Slot Surface-mounted Permanent Magnet Brushless Machines», IEEE Trans. on Energy Conversion, Vol. 28, No. 6, 2009. 
El-Refaie A. M., Jahns T.M., McCleer P. J., McKeever. J. W., «Experimental Verification of Optimal Flux Weakening in surface PM Machines Using Concentrated Windings», IEEE Trans. On Industry Applications, Vol. 42, No. 2, March/April 2006.

Espanet C., Modélisation et conception optimale de moteurs sans balais à structure inversée application au moteur-roue, Thèse de doctorat, Université de Franche-Comté, 1999.

Flux2D, Manuel d'utilisation, Cedrat.

Foggia A., Méthodes de calcul des inductances de fuites, Technique de l'Ingénieur, D3440, Feb. 1999.

Ishak D., Zhu Z., Howe D., «Permanent Magnet Brushless Machines with Unequal Tooth Widths and Similar Slot and Pole Numbers», IEEE Trans. on Energy Conversion, 2004.

Ishak D., Zhu Z., Howe D., « Comparison of PM Brushless Motors, Having Either All Teeth or Alternate Teeth Wound », IEEE Trans. on Energy Conversion, Vol. 21, No. 1, March 2006.

Jack A. G., Mecrow B.C. and Haylock J. A., «A Comparative Study of Permanent Magnet and Switched Reluctance Machines for High-Performance Fault-Tolerant Applications», IEEE Trans. Industry Applications, vol. 32, no. 4, 1996, pp. 889-895.

Libert F., Soulard J., «Investigation on Pole-Slot Combinations for Permament Magnet Machines with Concentrated Windings », Proceedings of the International Conference on Electrical Machines, ICEM 2004, Sept. 2004.

Mai H. C. M., Dubas F., Chamagne D., Espanet C., «Optimal Design of a Surface Mounted Permanent Magnet In-Wheel Motor for an Urban Hybrid Vehicle», In Proc. VPPC, IEEE Vehicle Power and Propulsion Conference, 7-11 Sep. 2009, Dearborn, USA.

Magnussen F., Lendenmann H., «Parasitic Effects in PM Machines With Concentrated Windings», IEEE Trans. On Industry Applications, Vol. 43, No. 5, Sep. /Oct. 2007.

Markovic M., Perriard Y., «An Analytical Determination of Eddy-Current Losses in a Configuration With a Rotating Permanent Magnet», IEEE Trans. On Magnetics, Vol. 43, No. 8, August 2007.

Mitcham A. J., Antonopoulos G. and Cullen J. J. A., «Favourable slot and pole number combinations for fault-tolerant PM machines», IEE Proc.-Electr. Power Appication., Vol. 151, No. 5, September 2004.

Multon B., Lucidarme J., Prévond L., « Analyse des possibilités de fonctionnement en régime de désexcitation des moteurs à aimants permanents », J. Phys. III France 5 (1995), May 1995, pp. 623-640.

Saint-Michel J., «Bobinage des machines tournantes à courant alternatif», Technique de l'Ingénieur, D 3420.

Salminen P., Niemelä M. and Pyrhönen J., « Performance analysis of fractional slot wound Pm motors for low speed applications », IEEE Industry Applications, 2004, pp. 10321037.

Slemon G., Liu X. «Core losses in permanent magnet motors», IEEE Trans. on Magnetic, Vol. 26, No. 5, Septembre 1990. 
Comparaison de deux types de bobinage pour la réalisation de moteur-roues à aimants permanents destinés à la propulsion d'un véhicule hybride urbain

Soong W. L., Ertugrul N., «Field-Weakening Performance of Interior Permanent-Magnet Motors», IEEE Trans. On Industry Apsplications, Vol. 38, No. 5, Sep. /Oct. 2002.

Zhu. Z.Q., Howe. D., Mitchell. J.K., « Magnetic field analysis and inductances of brushless DC machines with surface-mounted magnets and non-overlapping stator windings », IEEE Trans. on Magnetic, volume 31, no. 3, May 1995, pp. 2115 - 2118. 